\title{
A) ARTIGO ORIGINAL

\section{Fitonematoides associados à Zingiberales tropicais e estimativas do número de amostras para monitoramento}

\author{
Plant parasitic nematodes associated with tropical \\ Zingiberales and sample size estimates for monitoring
}

Tereza Cristina de Assis ${ }^{*}$, Elvira Maria Regis Pedrosa²,

Domingos Eduardo Guimarães Tavares de Andrade ${ }^{3}$, Rildo Sartori Barbosa Coelho ${ }^{4}$,

Luciana Melo Sartori Gurgel ${ }^{5}$, Regina Ceres Torres da Rosa ${ }^{6}$

'Laboratốrio de Fitossanidade, Instituto Agronômico de Pernambuco (IPA), Av. General San Martin, 1371, Bongi, CEP 50761-000

Recife, PE, Brasil

${ }^{2}$ Departamento de Agronomia, Universidade Federal Rural de

Pernambuco (UFRPE), Recife, PE, Brasil ${ }^{3}$ Instituto Agronômico de Pernambuco (IPA), Estação Experimental de Itapirema, Goiana, PE, Brasil ${ }^{4}$ Departamento de Pesquisa, Instituto Agronômico de Pernambuco (IPA), Recife, PE, Brasil

${ }^{5}$ Departamento de Negócios

Tecnológicos, Instituto Agronômico de Pernambuco (IPA), Recife, PE, Brasil

${ }^{6}$ Laboratốrio de Patologia de

Sementes, Instituto Agronômico de

Pernambuco (IPA), Recife, PE, Brasil

*autor correspondente

凶 cristina.assis@ipa.br
RESUMO: Este estudo teve por objetivos realizar o levantamento de fitonematoides associados a Zingiberales ornamentais (Etlingera elatior, Zingiber spectabilis, Alpinia purpurata, Musa coccinea, Tapeinoquilos ananassae e Heliconia spp.) em áreas produtoras de Pernambuco e estimar o número de amostras para monitoramento. Amostras de raízes de plantas infectadas e solo da rizosfera foram coletados em 10 áreas produtoras, no período de 2002 a 2004, e processadas pelo método de flotação centrífuga. As densidades populacionais dos gêneros presentes foram determinadas e utilizadas para estimar o número de amostras para monitoramento. Constatou-se ocorrência frequente dos seguintes gêneros: Pratylenchus, Rotylenchulus, Meloidogyne, Helicotylenchus e Criconemella. Sintomas da meloidoginose foram constatados em $100 \%$ das áreas de plantio, com níveis populacionais de Meloidogyne spp. alcançando 462 espécimes/300 $\mathrm{cm}^{3}$ de solo e 7.452 espécimes/20 g de raízes. As espécies de Meloidogyne detectadas foram $M$. incognita, M. arenaria e M. javanica. Para monitoramento dos gêneros Pratylenchus e Meloidogyne, é recomendada a utilização de 11 e 10 amostras de raízes, respectivamente, considerando um nível de erro de $20 \%$. As associações M. arenaria com E. elatior, Rotylenchulus sp. com A. purpurata, Heliconia spp. e M. coccinia, e Criconemella sp. em A. purpurata constituíram os primeiros relatos da ocorrência em Pernambuco.

PALAVRAS-CHAVE: Heliconia spp., Etlingera elatior, Alpinia purpurata, Zingiber spectabilis, Musa coccinea, Tapeinoquilos ananassae.
ABSTRACT: The purpose of this study was to screen plant parasitic nematodes in Zingiberales (Etlingera elatior, Zingiber spectabilis, Alpinia purpurata, Musa coccinea, Tapeinoquilos ananassae and Heliconia spp.) producing areas of the State of Pernambuco and estimate sample size for nematodes monitoring. Samples (soil and roots of nematode parasitized plants) were collected in 10 producing areas, from 2002 to 2004 and processed by centrifugal flotation method. Population density of plant parasitic nematodes were evaluated and used to estimate sample size for monitoring. Pratylenchus sp., Rotylenchulus sp., Meloidogyne spp., Helicotylenchus sp. and Criconemella sp. were frequent in most of samples. Symptoms of root-knot nematodes were verified in $100 \%$ of the planting areas, with population levels of Meloidogyne spp. up to 462 nematodes per $300 \mathrm{~cm}^{3}$ of soil and 7,452 nematodes per $20 \mathrm{~g}$ of roots. Among Meloidogyne species, M. incognita, M. arenaria and M. javanica were detected. Data analysis indicated that at least 11 and 10 samples of root are recommended for monitoring the genus Pratylenchus and Meloidogyne, respectively, at $20 \%$ error level. The associations M. arenaria with E. elatior, Rotylenchulus sp. with A. purpurata, Heliconia spp. and M. coccinia, and Criconemella sp. with A. purpurata were the first occurrence reported in the State of Pernambuco, Brazil.

KEYWORDS: Heliconia spp., Etlingera elatior, Alpinia purpurata, Zingiber spectabilis, Musa coccinea, Tapeinoquilos ananassae. 


\section{Introdução}

A floricultura no Brasil é uma atividade consolidada, sendo o Estado de Pernambuco o maior produtor de flores tropicais do País com cerca de 200 produtores participando na produção e comercialização das flores. Nesse Estado são movimentados recursos da ordem de $\mathrm{R} \$ 36$ milhões/ano que geram em torno de 800 empregos diretos e muitos indiretos (BRASIL, 2012). O aumento expressivo em área plantada com ornamentais tropicais no Estado, ocorrido nos últimos 15 anos, está concentrado nas regiões do Litoral e da Mata, distribuídos pelos municípios de Camaragibe, Paulista, Ipojuca, Igarassu, Recife, Escada, Moreno, Cabo de Santo Agostinho, Ribeirão, Água Preta, Sairé, Vitória de Santo Antão e Petrolina (BRASIL, 2007).

Apesar das muitas vantagens e oportunidades para cultivo de Zingiberales ornamentais, o parasitismo de nematoides pode causar perdas elevadas na produção (COELHO; WARUMBY, 2002). O nível de dano das fitonematoses depende de vários fatores, destacando-se a susceptibilidade da cultura, condições ambientais, densidade populacional dos fitonematoides e presença de outros organismos fitopatogênicos, que podem interagir com o fitoparasito (TIHOHOD, 2000). Além disso, a densidade populacional do parasito pode variar durante todo o ano nos climas tropicais em face às variações de temperatura e umidade do solo (LORDELLO, 1984).

As fitonematoses causadas por espécies dos gêneros Meloidogyne Göeldi, Radopholus Thorne, Helicotylenchus Steiner e Pratylenchus Filip'ev vêm constituindo um dos principais problemas fitossanitários em plantas ornamentais tropicais em Pernambuco (COELHO; WARUMBY, 2002; ASSIS, 2006). O parasitismo tem ocorrência comum em alpínias (Alpinia spp.), bastão do imperador (Etlingera elatior R.M. Smith), musas (Musa spp.) e helicônias (Heliconia spp.), causando danos às raízes e, consequentemente, redução severa no crescimento e morte de plantas (ASSIS, 2006). O controle das fitonematoses é difícil e deve envolver diversas estratégias para reduzir a população a níveis que não afetem o desenvolvimento da planta, sendo fundamental o monitoramento das populações presentes.

Apesar da importância das fitonematoses para as Zingiberales ornamentais, não existem levantamentos sobre a intensidade da doença em áreas produtoras, nem sobre o número de amostras recomendado para monitoramento das populações presentes. Levantamentos fitopatológicos são fundamentais para obtenção de informações sobre a importância relativa de doenças, o nível geral de sanidade das culturas, presença ou ausência de doenças quarentenárias, monitoramento de flutuações populacionais dos fitopatógenos ao longo do tempo e análise de eficiência de práticas de controle recomendadas (HOLDERNESS, 2002), constituindo-se em importante instrumento para o desenvolvimento de programas de manejo integrado de doenças.

$\mathrm{O}$ número de amostras, tomado em um experimento ou levantamento de campo, determina a qualidade ou a confiabilidade dos dados de quantificação da doença obtidos e o custo da iniciativa. No entanto, é necessário o ajuste entre o que é biológica e estatisticamente aceitável (MADDEN; HUGHES; BOSCH, 2007). O presente trabalho teve por objetivos efetuar levantamento de fitonematoides associados a Zingiberales ornamentais tropicais em áreas de plantio do Estado de Pernambuco e estimar o número de amostras recomendado para monitorar populações de fitonematoides em futuros levantamentos.

\section{Material e Métodos}

\section{Levantamento de fitonematoides em Zingiberales ornamentais}

No período de 2002 a 2004, foram realizados levantamentos de fitonematoides em 10 áreas produtoras das seguintes Zingiberales ornamentais: bastão do imperador, zingiber (Zingiber spectabilis Roscoe), alpínia (Alpinia purpurata ((Vieill.) Schum.), musa ornamental (Musa coccinea Andrews), tapeinóquilos (Tapeinoquilos ananassae Hassk) e helicônias (Heliconia spp.), localizadas nos municípios de Igarassu, Ribeirão, Cabo de Santo Augostinho, Alecrim, Jaboatão dos Guararapes, Paudalho e São Lourenço da Mata, do Estado de Pernambuco (Tabela 1). As áreas de produção foram tomadas ao acaso e as coletas realizadas em plantas doentes que se encontravam em diferentes estágios de desenvolvimento (vegetativo e floração).

Em cada propriedade, foram coletadas raízes de plantas parasitadas por fitonematoides e solo de rizosfera, com número de amostras $(\mathrm{n})$ variável por área, onde $\mathrm{n} \geq$ oito amostras. Informações adicionais sobre o local e a espécie de Zingiberales envolvida foram obtidas de cada área. Depois da coleta, o material devidamente acondicionado e etiquetado foi processado no Laboratório de Fitonematologia da Universidade Federal Rural de Pernambuco-UFRPE.

O processamento das amostras de solo foi realizado pelo método de flotação centrífuga (JENKINS, 1964). Para a extração de nematoides das raízes, $100 \mathrm{~g}$ de raízes foram lavadas, cortadas em pedaços de aproximadamente $2 \mathrm{~cm}$ e trituradas em liquidificador, em baixa velocidade, por 10 a 20 segundos. O macerado mais o líquido foram passados em peneiras de 60 sobre 400 meshes, e o filtrado foi observado diretamente em microscópio ou, quando necessário, processado pelo método de Jenkins (1964).

A avaliação consistiu na identificação dos gêneros de fitonematoides presentes, segundo chave de identificação de Mai e Mullin (1996) e na determinação da densidade populacional de cada gênero identificado. A identificação de espécies de Meloidogyne fundamentou-se no modelo perineal de fêmeas adultas, segundo Taylor e Sasser (1978), sendo utilizada a metodologia de Taylor e Netscher (1974) no preparo dos corantes e das lâminas.

A prevalência dos fitonematoides foi estimada pela quantidade de áreas com presença do nematoide em relação ao total de áreas analisadas (MADDEN; HUGHES; BOSCH, 2007).

\section{Determinação do número de amostras para monitoramento de fitonematoides em Zingiberales}

Os dados obtidos no levantamento foram analisados conforme a metodologia descrita por Campbell e Madden (1990). O 
Tabela 1. Espécies de Zingiberales ornamentais coletados em municípios do Estado de Pernambuco.

\begin{tabular}{|c|c|c|}
\hline Áreas & Município & Espécies coletadas \\
\hline A-01 & Igarassu & $\begin{array}{l}\text { Etlingera elatior, Zingiber spectabilis, Alpinia purpurata, Musa coccinea, Heliconia psittacorum } \\
\text { (var. Alan Carle e Saci) e H. stricta (var. Las Cruses) }\end{array}$ \\
\hline A-02 & Igarassu & E. elatior, H. psittacorum (var. Red Opol) e H. rostrata \\
\hline A-03 & Igarassu & $\begin{array}{l}\text { E. elatior, A. purpurata, M. coccinea, H. ortotricha (var. She), H. psittacorum (var. Red Opol e Gold Red } \\
\text { Adrian) e H. charteacea (var. Sexy Pink) }\end{array}$ \\
\hline A-04 & Ribeirão & E. elatior, A. purpurata, M. coccinea e H. psittacorum (var. Alan Carle) \\
\hline A-05 & Ribeirão & E. elatior, A. purpurata e H. psittacorum (var. Alan Carle) \\
\hline A-06 & Cabo de Santo Augostinho & Tapeinoquilos ananassae, A. purpurata, M. coccinea, H. rostrata (var. Grande) e H. stricta (var. Faribard) \\
\hline A-07 & Alecrim & A. purpurata e H.psittacorum (var. Alan Carle) \\
\hline A-08 & Jaboatão dos Guararapes & E. elatior, A. purpurata e M. coccinea \\
\hline A-09 & Paudalho & E. elatior e H. birrai \\
\hline A-10 & São Lourenço da Mata & A. purpurata e $M$. coccinea \\
\hline
\end{tabular}

número de amostras (n) para monitoramento foi estimado para cada área onde os fitonematoides Meloidogyne spp. e Pratylenchus sp. foram observados, utilizando-se a equação: $\mathrm{n}=\mathrm{S}^{2} /\left(\mathrm{X}^{2} \cdot \mathrm{CVX}^{2}\right)$, na qual $\mathrm{X}$ correspondeu às médias populacionais do fitonematoide; $\mathrm{S}^{2}$ variância da média; e CVx ao coeficiente de variação da média, considerando-se confiabilidades (erros aceitáveis) pré-estabelecidas de 5\%, $10 \%, 15 \%$ e $20 \%(\mathrm{CVx}=0,05,0,1,0,15$ e 0,20$)$. Utilizando-se os dados obtidos para cada área, foi calculado o número ideal médio de amostras para monitoramento, considerando os diferentes níveis de erro aceitáveis.

Visando comparar a influência dos níveis populacionais dos fitonematoides sobre o número de amostras, foi efetuada a análise de correlação de Pearson, ao nível de 5\% de probabilidade.

\section{Resultados e Discussão}

\section{Levantamento de fitonematoides em Zingiberales ornamentais}

No levantamento efetuado em áreas de cultivo de Zingiberales ornamentais, no período de 2002 a 2004, constatou-se a ocorrência dos seguintes gêneros de fitonematoides: Pratylenchus, Rotylenchulus Filip'ev, Meloidogyne, Helicotylenchus e Criconemella De Grisse \& Loof, sendo a densidade populacional variável entre os diferentes hospedeiros avaliados (Tabela 2). Em Pernambuco, esses gêneros de fitonematoides já foram relacionados em Zingiberales (COELHO, 2001), com exceção de Rotylenchulus e Criconemella, que não constam como parasitos de plantas da ordem Zingiberales. Desde a década de 90, no entanto, é relatado no Havaí Rotylenchulus reniformis Lindford \& Oliveira parasitando Heliconia spp. (SEWAKE; UCHIDA, 1995), enquanto Criconemella onoensis Luc foi relatado na América Central parasitando Alpinia sp. (BALA; HOSEIN, 1996). Não foi verificada a presença de Radopholus, outro importante gênero de fitonematoide previamente relatado por Coelho e Warumby (2002).

Os níveis populacionais médios para Meloidogyne spp. nos diferentes hospedeiros foram elevados, variando de 0 a 374,7 espécimes/300 $\mathrm{cm}^{3}$ de solo e de 208,8 a 6089,9 espécimes/20 g de raízes; seguido de Rotylenchulus sp. (0 a 132,3 espécimes/300 $\mathrm{cm}^{3}$ de solo e de 0 a 402,1 espécimes/20 g de raízes); Helicotylenchus sp. (15,7 a 106,6 espécimes $/ 300 \mathrm{~cm}^{3}$ de solo e de 0 a 165,9 espécimes $/ 20 \mathrm{~g}$ de raízes); Pratylenchus sp. (0 a 29,7 espécimes $/ 300 \mathrm{~cm}^{3}$ de solo e de 0 a 47,9 espécimes/20 g de raízes); e Criconemella sp. com baixos níveis populacionais (0 a 5,8 espécimes $/ 300 \mathrm{~cm}^{3}$ de solo e de 0 a 39,0 espécimes/20 g de raízes) (Tabela 2). De forma similar, Bala e Hosein (1996) em levantamentos realizados em Trinidad e Tobago observaram elevada frequência de $M$. incognita (Kofoit \& White) Chitwood em Alpinia sp e Heliconia sp., que, segundo os autores, contribuiu para o menor crescimento das plantas e, consequente redução da produção de flores.

Estudos morfológicos da região perineal de fêmeas adultas identificaram o parasitismo das espécies $M$. incognita, M. arenaria (Neal) Chitwood e $M$. javanica (Treub) Chitwood, as quais incitaram sintomas reflexos como nanismo, caules finos, desfolhamento, amarelecimento, enrolamento e necrose das folhas, flores pequenas e descoloridas; e diretos, a exemplo de sistema radicular com poucas raízes e com formação de galhas, em todas as espécies de plantas analisadas neste levantamento (Figura 1). As espécies $M$. incognita e $M$. javanica são relatadas como importantes causadoras de galhas em helicônias (TANGONAN; QUEBRAL, 1992; FREIRE; MOSCA, 2009), no entanto não foram encontrados relatos na literatura especializada para $M$. arenaria, sendo este, portanto, o primeiro relato de ocorrência em Zingiberales ornamentais.

As associações parasíticas de M. arenaria em E. elatior; Rotylenchulus sp. em A. purpurata, Heliconia spp. e $M$. coccinia; e, Criconemella sp. em A. purpurata constituíram os primeiros relatos da ocorrência desses fitonematoides em 

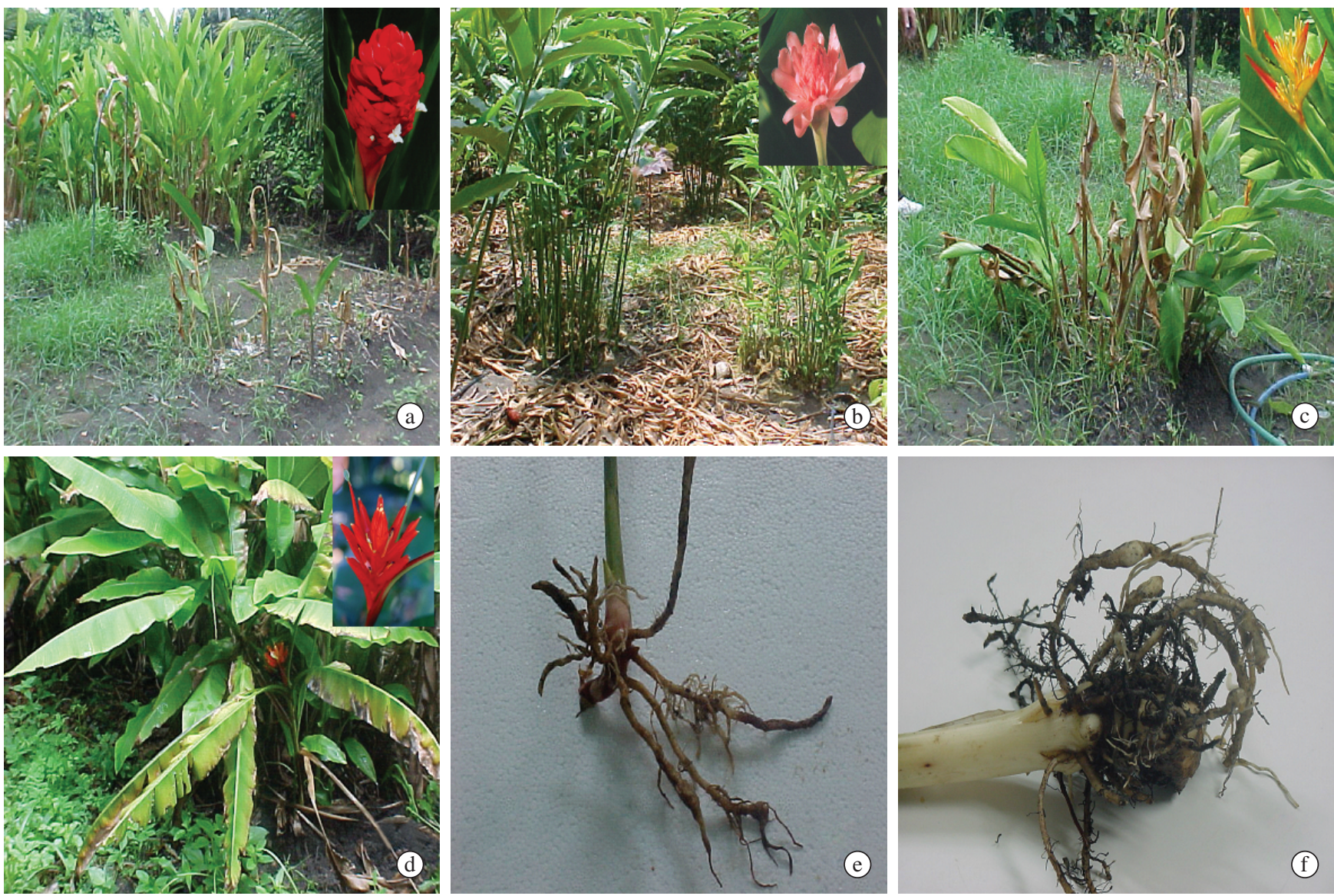

Figura 1. Sintomas causados por Meloidogyne spp. em Alpinia purpurata (a) e e)), Etlingera elatior (b) e f)), Heliconia spp. (c) e Musa coccinia (d).

Tabela 2. Densidade populacional de fitonematoides associados a diferentes Zingibelares tropicais no Estado de Pernambuco.

\begin{tabular}{|c|c|c|c|c|c|}
\hline \multirow{2}{*}{ Hospedeiro } & \multicolumn{5}{|c|}{ Densidade populacional $^{1}$} \\
\hline & Pratylenchus & Rotylenchulus & Meloidogyne & Helicotylenchus & Criconemella \\
\hline \multicolumn{6}{|l|}{ Solo } \\
\hline Alpinia purpurata & 2,7 & 37,5 & 148,9 & 30,3 & 5,8 \\
\hline Etlingera elatior & 29,7 & 59,2 & 135,8 & 15,7 & 0,0 \\
\hline Heliconia spp. ${ }^{2}$ & 4,7 & 45,5 & 106,9 & 18,0 & 0,0 \\
\hline Musa coccínea & 4,9 & 132,3 & 374,7 & 40,4 & 0,0 \\
\hline Tapeinochilos ananassae & 0,0 & 70,6 & 0,0 & 106,6 & 0,0 \\
\hline Zingiber spectabilis & 0,0 & 0,0 & 0,0 & 17,6 & 0,0 \\
\hline \multicolumn{6}{|l|}{ Raízes } \\
\hline Alpinia purpurata & 24,4 & 402,1 & 3904,2 & 28,4 & 39,0 \\
\hline Etlingera elatior & 22,5 & 0,0 & 6089,9 & 27,5 & 0,0 \\
\hline Heliconia spp. $^{2}$ & 2,9 & 4,0 & 208,8 & 0,8 & 0,0 \\
\hline Musa coccínea & 47,9 & 56,7 & 3047,8 & 165,9 & 0,0 \\
\hline Tapeinochilos ananassae & 0,0 & 0,0 & 412,3 & 79,0 & 0,0 \\
\hline Zingiber spectabilis & 0,0 & 0,0 & 622,1 & 0,0 & 0,0 \\
\hline
\end{tabular}

${ }^{1}$ Densidade populacional média: no solo (nível populacional médio de fitonematoides avaliado em amostras de $300 \mathrm{~cm}^{3}$ de solo); nas raízes (nível populacional médio de fitonematoides avaliado em $20 \mathrm{~g}$ de raízes); ${ }^{2}$ Heliconia psittacorum, $H$. stricta, $H$. rostrata, $H$. ortotricha, $H$. chartacea, $H$. birai e $H$. psittacorum $x$ H. spathocircinata. 
Tabela 3. Densidade populacional de fitonematoides associados a Zingiberales ornamentais e respectivas áreas de estudo no Estado de Pernambuco.

\begin{tabular}{|c|c|c|c|c|c|}
\hline \multirow{2}{*}{ Áreas } & \multicolumn{5}{|c|}{ Densidade populacional $^{1}$} \\
\hline & Pratylenchus & Rotylenchulus & Meloidogyne & Helicotylenchus & Criconemella \\
\hline \multicolumn{6}{|l|}{ Solo } \\
\hline A-01 & 5,89 & 40,44 & 22,66 & 38,45 & - \\
\hline A-02 & 8,88 & 59,11 & 231,02 & 56,17 & - \\
\hline A-03 & 3,52 & 157,28 & 47,97 & 46,10 & 0,88 \\
\hline A-04 & - & 13,26 & 51,06 & 4,40 & - \\
\hline A-05 & - & 4,40 & 124,40 & 31,06 & 4,40 \\
\hline A-06 & - & 36,68 & 462,13 & 30,47 & - \\
\hline A-07 & - & - & 346,57 & 8,80 & - \\
\hline A-08 & 38,00 & 235,53 & 215,33 & - & 11,33 \\
\hline A-09 & 40,00 & 13,33 & 193,33 & 16,66 & - \\
\hline A-10 & - & 53,33 & 113,33 & 20,00 & 6,66 \\
\hline Média & 9,63 & 61,34 & 180,78 & 25,21 & 2,33 \\
\hline \multicolumn{6}{|l|}{ Raízes } \\
\hline A-01 & 5,62 & 8,38 & 799,87 & 14,05 & - \\
\hline A-02 & - & - & $6.069,71$ & 50,79 & - \\
\hline A-03 & 55,84 & 1,25 & $1.455,31$ & 21,13 & - \\
\hline A-04 & 41,23 & 3,14 & $1.123,71$ & 15,80 & - \\
\hline A-05 & - & - & $2.762,50$ & 23,80 & 126,91 \\
\hline A-06 & - & - & $2.376,05$ & 163,12 & - \\
\hline A-07 & - & - & $6.856,82$ & - & - \\
\hline A-08 & - & $1.293,57$ & $7.452,38$ & 110,95 & - \\
\hline A-09 & 55,47 & 11,90 & $4.765,71$ & - & - \\
\hline A-10 & - & 238,09 & 869,76 & 23,80 & - \\
\hline Média & 15,82 & 155,63 & $3.453,18$ & 42,34 & 12,69 \\
\hline
\end{tabular}

'Densidade populacional média: no solo (nível populacional médio de fitonematoides avaliado em amostras de $300 \mathrm{~cm}^{3}$ de solo); nas raízes (nível populacional médio de fitonematoides avaliado em $20 \mathrm{~g}$ de raízes).

áreas comerciais do Estado de Pernambuco. Neste estudo, destaque deve ser dado também às elevadas populações de M. incognita observadas em A. purpurata (var. Rosa, Kimi, Vermelha e Jungle King), H. psittacorum (var Alan Carle, Red Opol, Saci, Gold Red Adrian), H. stricta (var Las cruces), $H$. rostrata, H. ortotricha (var She), H. chartacea (var sexy pink), $M$. coccinea, e em associação com $M$. arenaria e M. javanica em E. elatior.

Sintomas de meloidoginose foram constatados em 100\% das áreas de plantio, indicando alta prevalência da doença nos municípios analisados, seguido de Rotylenchulus e Helicotylenchus constatados em $90 \%$ das áreas e, em menor prevalência, Pratylenchus e Criconemella, respectivamente ocorrendo em $50 \%$ e $40 \%$ das áreas estudadas (Tabela 3). A densidade populacional entre os gêneros de fitonematoides variou quando analisado conjuntamente às áreas de plantio. Considerando amostras de solo e raiz, a densidade populacional média de Meloidogyne spp. (180,78 espécimes $/ 300 \mathrm{~cm}^{3}$ de solo e 3453,18 espécimes/20 g de raiz) foi superior a dos demais fitonematoides. Fato semelhante foi verificado por Costa et al. (2001), em que espécies do gênero Meloidogyne predominaram em relação às demais, nas associações com plantas ornamentais de diferentes gêneros.

Em 80\% das áreas, foram constatados níveis populacionais extremamente elevados de Meloidogyne spp., variando entre 1123,71 e 7452,38 espécimes/20 g de raízes, enquanto que, em apenas $20 \%$ das áreas, observaram-se densidades inferiores, variando de 799,87 a 869,76 (Tabela 3). Os demais fitonematoides apresentaram níveis populacionais relativamente baixos quando comparados com Meloidogyne spp., entretanto a manutenção de plantas infectadas no campo pode propiciar a elevação dos níveis populacionais ao longo do tempo (FREITAS; OLIVEIRA; FERRAZ, 2009). Outro fator preocupante é a comercialização de mudas infectadas por fitonematoides, as quais são fontes de inóculo primário para áreas em que este ainda não tenha ocorrido.

A meloidoginose encontra-se generalizada nas áreas de plantio dos municípios visitados do Estado de Pernambuco, 
indicando uma grande adaptação do fitonematoide ao ambiente e aos hospedeiros. Os elevados níveis populacionais de Meloidogyne spp. no solo e raízes de Zingiberales verificados neste estudo podem inviabilizar, num futuro próximo, o cultivo destas ornamentais, por promover redução do crescimento das plantas e, consequentemente, baixíssima produção e/ou produtividade, caso medidas de controle não sejam empregadas no manejo da doença.

\section{Determinação do número de amostras para} monitoramento de fitonematoides em Zingiberales

$\mathrm{Na}$ estimativa do número ideal de amostras para quantificação das populações dos fitonematoides em áreas de cultivo, o número de amostras a serem consideradas reduziu significativamente $(\mathrm{P} \leq 0,05)$ com a elevação do erro aceitável (Tabela 4). Considerando-se a média de 10 áreas de plantio e assumindo um elevado rigor no levantamento (5\% de erro), 179 e 174 amostras de $20 \mathrm{~g}$ de raízes/área, respectivamente para Pratylenchus e Meloidogyne, são apropriadas para quantificar as populações destes fitonematoides. No entanto, a escolha do nível de erro a ser utilizado na determinação do número ideal de amostras, depende do propósito da amostragem (KRANZ, 1988) e da relação custo/benefício envolvida no levantamento (MADDEN; HUGHES; BOSCH, 2007). Portanto, em futuros levantamentos de fitonematoides, em cada área de cultivo devem ser coletadas 10 e 11 amostras de raízes por área de 1 a 3 ha, respectivamente para Meloidogyne e Pratylenchus, admitindo um nível de erro máximo de $20 \%$, percentual este considerado adequado em levantamentos de campo (SOUTHWOOD, 1978).

As diferenças entre os números de amostras de solo e raízes necessários para quantificar as populações do mesmo fitonematoide podem ser explicadas pela variação entre as médias e as variâncias observadas entre as áreas de cultivo de zingiberales ornamentais. Neste estudo, não foram verificadas correlações significativas entre as densidades populacionais dos fitonematoides e o número de amostras para monitoramento, como verificado em outros patossistemas por Rossi e Battilani (1989) e Jong (1995).

Um pressuposto básico para estimar o número de amostras é que os dados dos locais analisados são representativos do que poderia ocorrer em outros campos, sendo a validade

Tabela 4. Estimativa da quantidade de amostras de raízes necessárias para monitoramento de fitonematoides em zingiberales tropicais, com a confiabilidade definida pelo coeficiente de variação da média (erro).

\begin{tabular}{|c|c|c|c|c|c|c|}
\hline \multirow{2}{*}{ Espécie/Área } & \multirow{2}{*}{ Densidade populacional $^{1}$} & \multirow{2}{*}{ Variância $^{2}$} & \multicolumn{4}{|c|}{$\operatorname{Erro}(\%)^{3}$} \\
\hline & & & 5 & 10 & 15 & 20 \\
\hline \multicolumn{7}{|l|}{ Pratylenchus } \\
\hline A-01 & 5,6 & 28,2 & 356,4 & 89,1 & 39,6 & 22,3 \\
\hline A-02 & 0,0 & 0,0 & 0,0 & 0,0 & 0,0 & 0,0 \\
\hline A- -03 & 55,8 & 1527,6 & 195,9 & 49,0 & 21,8 & 12,2 \\
\hline A-04 & 41,2 & 3525,2 & 829,2 & 207,3 & 92,1 & 51,8 \\
\hline A- -05 & 0,0 & 0,0 & 0,0 & 0,0 & 0,0 & 0,0 \\
\hline A-06 & 0,0 & 0,0 & 0,0 & 0,0 & 0,0 & 0,0 \\
\hline A- -07 & 0,0 & 0,0 & 0,0 & 0,0 & 0,0 & 0,0 \\
\hline A-08 & 0,0 & 0,0 & 0,0 & 0,0 & 0,0 & 0,0 \\
\hline A-09 & 55,5 & 3184,4 & 413,9 & 103,5 & 46,0 & 25,9 \\
\hline A-10 & 0,0 & 0,0 & 0,0 & 0,0 & 0,0 & 0,0 \\
\hline Média & 15,8 & - & 179,5 & 44,9 & 19,9 & 11,2 \\
\hline \multicolumn{7}{|l|}{ Meloidogyne } \\
\hline A-01 & 799,9 & 80537,7 & 50,4 & 12,6 & 5,6 & 3,1 \\
\hline A-02 & 6069,7 & 1073160,6 & 11,7 & 2,9 & 1,3 & 0,7 \\
\hline A-03 & 1455,3 & 680492,9 & 128,5 & 32,1 & 14,3 & 8,0 \\
\hline A-04 & 1123,7 & 2078783,1 & 658,5 & 164,6 & 73,2 & 41,2 \\
\hline A-05 & 2762,5 & 929918,3 & 48,7 & 12,2 & 5,4 & 3,0 \\
\hline A- -06 & 2376,1 & 1855432,1 & 131,5 & 32,9 & 14,6 & 8,2 \\
\hline A-07 & 6856,8 & 6570142,2 & 55,9 & 14,0 & 6,2 & 3,5 \\
\hline A-08 & 7452,4 & 3913756,1 & 28,2 & 7,0 & 3,1 & 1,8 \\
\hline A-09 & 4765,7 & 35776800,9 & 630,1 & 157,5 & 70,0 & 39,4 \\
\hline A-10 & 869,8 & 7257,3 & 3,8 & 1,0 & 0,4 & 0,2 \\
\hline Média & 3453,2 & - & 174,7 & 43,7 & 19,4 & 10,9 \\
\hline
\end{tabular}

${ }^{1}$ Média populacional do fitonematoide em áreas de plantio do Estado de Pernambuco. ${ }^{2}$ Variância $\left(\mathrm{S}^{2}\right)$ média da população de fitonematoides em áreas de plantio do Estado de Pernambuco. ${ }^{3}$ Tamanho ideal da amostra admitindo erros de 5, 10, 15 e 20, conforme Campbell e Madden (1990). 
desses pressupostos variável entre patossistemas (MADDEN; HUGHES; BOSCH, 2007). Outro aspecto importante a considerar é que o número de amostras para quantificar as populações de fitonematoides precisa ser dinâmico, uma vez que pode variar com o progresso da doença (KRANZ, 1988; DUTHIE; CAMPBELL; NELSON, 1991) e com as mudanças do arranjo espacial de plantas doentes no campo durante o desenvolvimento da doença (KRANZ, 1988; CAMPBELL; DUTHIE, 1989).

\section{Conclusões}

Os gêneros Pratylenchus, Rotylenchulus, Meloidogyne, Helicotylenchus e Criconemella foram frequentemente encontrados parasitando raízes de Zingiberales nas áreas de produção das zonas da Mata e Litoral do Estado de Pernambuco. A meloidoginose provocada por Meloidogyne incognita, M. arenaria e M. javanica foi constatada em todas as áreas de plantio visitadas, provocando visível diminuição das plantas e, consequentemente, perdas na produção e qualidade das flores, sendo esta situação extremamente preocupante para os produtores do Estado. No monitoramento populacional dos gêneros Pratylenchus e Meloidogyne, é recomendada a utilização de 11 e 10 amostras de raízes, respectivamente, considerando um nível de erro de $20 \%$. Os resultados deste estudo servem como base para futuros levantamentos de fitonematoses em Zingiberales no Estado de Pernambuco, uma vez que os dados foram originados de campos sob diferentes condições e estimados considerando necessidades crescentes de precisão.

\section{Referências}

ASSIS, T. C. Fitonematoides associados a Zingiberales ornamentais em Pernambuco: estimativa do número de amostras para monitoramento, efeito de indutores de resistência e avaliação de mecanismos envolvidos. 2006. 84 f. Tese (Doutorado em Fitopatologia)-Universidade Federal Rural de Pernambuco, Recife, 2006.

BALA, G.; HOSEIN, F. Plant-parasitic nematodes associated with anthuriums and other tropical ornamentals. Nematropica, Florida, v. 26, p. 9-14, 1996.

BRASIL. Ministério da Agricultura, Pecuária e Abastecimento. In: BUAINAIN, A. M.; BATALHA, M. O. (Coords.). Cadeia produtiva de flores e mel. Brasília: IICA, MAPA, SPA, 2007. 140 p.

BRASIL. Ministério do Desenvolvimento, Indústria e Comércio Exterior. Aprendendo a exportar flores 2012. Brasília, 2012. Disponível em: <http://www.aprendendoaexportar.gov.br/flores>. Acesso em: 15 fev. 2012.

CAMPBELL, C. L.; DUTHIE, J. A. Sampling for disease assessment. Biological and Cultural Tests for Control of Plant Diseases, v. 4, p. 5-8, 1989.

CAMPBELL, C. L.; MADDEN, L. V. Introduction to plant disease epidemiology. New York: John Wiley \& Sons, 1990. 532 p.
COELHO, R. S. B. Laudos da seção de fitossanidade: diagnóstico fitossanitário 2001. Recife: Empresa Pernambucana de Pesquisa Agropecuária-IPA, 2001. 15 p.

COELHO, R. S. B.; WARUMBY, J. F. Doenças de plantas ornamentais tropicais detectadas na Zona da Mata de Pernambuco. Floricultura em Pernambuco, v. 1, p. 67-69, 2002.

COSTA, M. J. N. et al. Nematóides em plantas ornamentais. Ciências Agrotécnicas, v. 25, p. 1127-1132, 2001.

DUTHIE, J. A.; CAMPBELL, C. L.; NELSON, L. A. Efficiency of multistage sampling for estimating of intensity of leaf spot diseases of alfafa in field experiments. Phytopathology, v. 81, p. 959-964, 1991. http://dx.doi.org/10.1094/Phyto-81-959

FREIRE, F. C. O.; MOSCA, J. L. Patógenos associados a doenças de plantas ornamentais no Estado do Ceará. Revista Brasileira de Horticultura Ornamental, v. 15, n. 1, p. 83-89, 2009.

FREITAS, L. G.; OLIVEIRA, R. D. L.; FERRAZ, S. Introdução à nematologia. Viçosa: Universidade Federal de Viçosa, 2009. 90 p.

HOLDERNESS, M. Surveys and sampling. In: WALLER, J. M.; LENNÉ, J. M.; WALLER, S. J. (Eds.). Plant pathologist's pocketbook. 3. ed. New York: CAB International, 2002. p. 19-24.

JENKINS, W. R. A. A rapid centrifugal-flotation technique for separating nematodes from soil. Plant Disease Report, v. 48, p. 692, 1964.

JONG, P. D. Sampling for detection: leek rust as a example. International Journal of Pest Management, v. 41, p. 31-35, 1995. http://dx.doi.org/10.1080/09670879509371918

KRANZ, J. Measuring plant disease. In: KRANZ, J.; ROTEM, J. (Eds.). Experimental techniques in plant disease epidemiology. Heidelberg: Springer-Verlag, 1988. p.35-50. http://dx.doi. org/10.1007/978-3-642-95534-1_4

LORDELLO, L. G. E. Nematóides das plantas cultivadas. 8. ed. São Paulo: Nobel, 1984. 314 p.

MADDEN, L. V.; HUGHES, G.; BOSCH, F. The study of plant disease epidemics. St. Paul: APS Press, 2007. 432 p.

MAI, W. F.; MULLIN, P. G. Plant-parasitic nematodes a pictorial key to genoma. 5. ed. New York: Cornell University Press, 1996. 277 p.

ROSSI, V.; BATTILANI, P. Assessment of intensity of Cercospora disease on sugarbeeet. Journal of Phytopathology, v. 124, p. 63-66, 1989.

SEWAKE, K. T.; UCHIDA, J. Y. Diseases of heliconia in hawaii. Honolulu: Hawaii Institute of Tropical Agriculture and Human Resources, $1995.18 \mathrm{p}$.

SOUTHWOOD, T. R. E. Ecological methods. 2. ed. London: Chapman \& Hall, 1978. 524 p. http://dx.doi.org/10.1007/978-94009-5809-8

TANGONAN, N. G.; QUEBRAL, F. C. Host index of plant diseases in the Philippines. 2. ed. St. Davao: G \& G Bisling Printers, 1992. 273 p.

TAYLOR, A. L.; SASSER, J. N. Biology, identification and control of root-knot nematodes (Meloidogyne species). North Caroline: International Meloidogyne Project, 1978. $111 \mathrm{p}$.

TAYLOR, D. P.; NETSCHER, C. An improved technique for preparing perineal patterns of Meloidogyne spp. Nematologica, v. 20, p. 268-269, 1974. http://dx.doi.org/10.1163/187529274X00285

TIHOHOD, D. Nematologia agrícola aplicada. Jaboticabal: FUNEP-SP, 2000. 473 p. 